\title{
Strategy and Execution: Lessons Learned from the Public Sector
}

\author{
Ali M. Al-Khouri, ${ }^{1,2}$ \\ ${ }^{1}$ Director General, Emirates Identity Authority, Abu Dhabi, UAE \\ ${ }^{2}$ Professor of Identity and Security, British Institute of Technology and E-commerce, London, UK \\ Correspondence: Ali M. Al-Khouri, Director General, Emirates Identity Authority, Abu Dhabi, UAE. E-mail: \\ ali.alkhouri@emiratesid.ae
}

Received: August 6, 2014

Accepted: August 19, 2014

Online Published: September 25, 2014

doi:10.5539/ibr.v7n10p61

URL: http://dx.doi.org/10.5539/ibr.v7n10p61

\begin{abstract}
The concept of strategic management in the public sector has attracted great attention in the past two decades. Unfortunately, current research shows that more attention is given to strategy development phases but less to strategy implementation. In fact, there is little literature about public sector strategies in the existing body of knowledge. In this article, we provide a short case study of strategy implementation at one of the most successful public sector organizations in the United Arab Emirates. The Emirates Identity Authority (Emirates ID) is a renowned government agency with an international reputation for its strategic planning and execution. The content of this article covers the 2010-2013 strategy at Emirates ID. We attempt to briefly analyze how the strategy impacted the overall organizational performance at Emirates ID and also attempt to explain some underlying factors that contributed to the overall success of the strategy. Finally, we draw on some of the lessons learned from this strategy exercise in terms of the challenges faced and how they have been tackled.
\end{abstract}

Keywords: strategy development, strategy execution, strategy implementation, strategic management, national ID, balance scorecard, project management, lessons learned

\section{Introduction}

Strategy, in its broadest definition, is referred to the "set of plans and policies by which a company aims to gain advantages over its competitors" (Skinner, 1969, p. 139). Other pragmatic definitions of strategy describe it in terms of answering the questions "where to go" and "how to get there" (Eisenhardt, 1999). Although the concept of competition may sound irrelevant in a governmental context, globally competitive environments have pushed governments worldwide to compete head to head in overall governance and various developmental fields. As a result, the topic of strategy in the public sector has been gaining greater attention in the past few years. However, there is not enough literature around public sector strategies in the existing body of knowledge.

This is viewed to be of particular importance, specifically if we take into account the high failure rates of strategies worldwide. On average, most strategies deliver only $63 \%$ on their performance potential, and many executives put this figure at 50\% (Mankins \& Steele, 2005). Figure 1 depicts the specific places where strategic planning and execution break down.

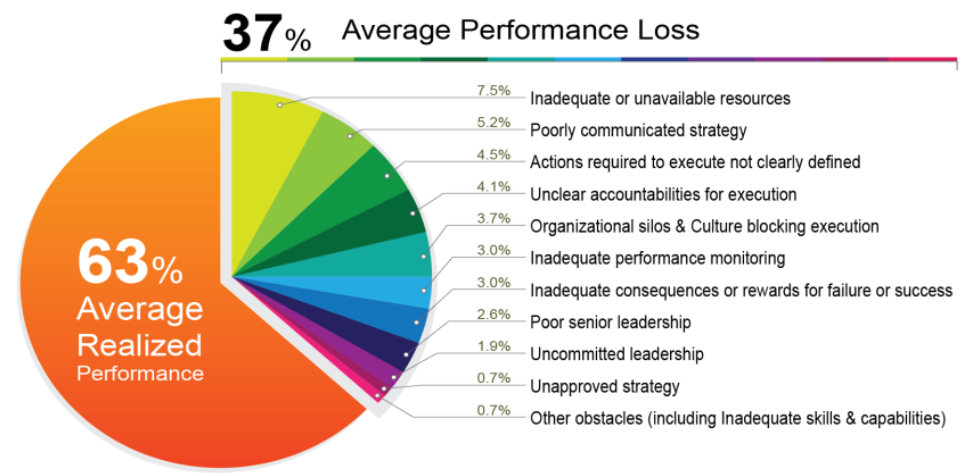

Figure 1. Strategy breakdowns

Source: Mankins \& Steele (2005). 
Carroll and Mui (2005) have researched 750 of the biggest business failures in the last 25 years and found that $46 \%$ of the catastrophes stemmed from misguided strategies. This shows that the single most acute cause of business failure has been a misguided or wrong strategy. Although there cannot be a right or wrong approach (i.e., what has worked for one organization may not necessarily work for another), we are of the belief that unbiased, published case studies will support the development of knowledge in this critical field of practice. This is where our article comes into play.

The focus of this article is to provide insights into a strategy development effort at one of the most successful public sector organizations in the United Arab Emirates. It explains the organization's overall strategy as well as how it achieved its set goals and objectives. The overview covers the 4-year strategy cycle of 2010-2013 and attempts to briefly analyze how the strategy impacted overall organizational performance. It also explains some of the underlying factors that have contributed to the overall success of the strategy and draws some lessons learned in terms of the challenges faced and how they were overcome.

This article is structured as follows. In Section 2, we briefly explain the research methodology we followed for this article. In Section 3, we provide a short overview about the organization being researched. In Section 4, we describe the 2010-2013 strategy of the Emirates Identity Authority. In Sections 5 and 6, we explain the strategy's objectives and how they impacted the organization's overall performance. In Section 7, we provide some foundational learned lessons, and we also explain the challenges and how the solutions were implemented. In Section 8 , we conclude the article.

\section{Research Methodology}

The purpose of this study is to provide a short case study of a strategy implementation at a government organization. The research approach followed in this article is a case study design. The reason for choosing a case study research design was because it is recognized as an ideal tool when a holistic, in-depth investigation is needed for investigating trends and specific situations (Feagin et al., 1991). Moreover, researchers have argued that certain kinds of information can be difficult, or even impossible, to tackle by means other than qualitative approaches, such as case study-based strategies (Sykes, 1990).

The selection of the organization was based on two issues: accessibility and its renowned international reputation for its strategic planning and execution as well as the alignment of strategy with organizational goals and objectives. The research design for this study is a descriptive and interpretive case study that is analyzed through qualitative methods. Data collection involved secondary sources (e.g., publications and technical documentation analysis) and primary sources (e.g., the active role of the author in the organization that facilitated contact with social actors).

\section{Emirates Identity Authority: Overview}

The Emirates ID Authority (Emirates ID) is a federal government organization in the United Arab Emirates. It began its journey in 2004 as a federal juridical government body with an independent budget and its own legal framework to facilitate achieving its objectives. The decree empowered the authority to develop and implement a sophisticated and state-of-art nationwide identity management infrastructure. The scheme involves enrolling the entire UAE population, including citizens and legal residents, and issuing them with unique identification numbers and smart cards that are linked with their biographical and biometric details.

\section{2010-2013 Strategy: Definition \& Aspirations}

In the definition of Emirates ID's strategic direction for 2010-2013, there was a distinct aim for national and individual security, the simplification of transactions, and service excellence, with an immediate requirement for Emirates ID to fulfill its mandate to enroll $100 \%$ of the residents and citizens of the United Arab Emirates who would serve to drive the near-term strategic focus. Considering the fact that Emirates ID was rapidly approaching a turning point in the evolution of its operating model, the strategy had to cater to the progression to the next level of identity sophistication, such as the support of the simplification of transitions and facilitation of innovative e-services. Figure 2 depicts the business operating model developed at Emirates ID that illustrates the expected transition in the organization's value chain. 


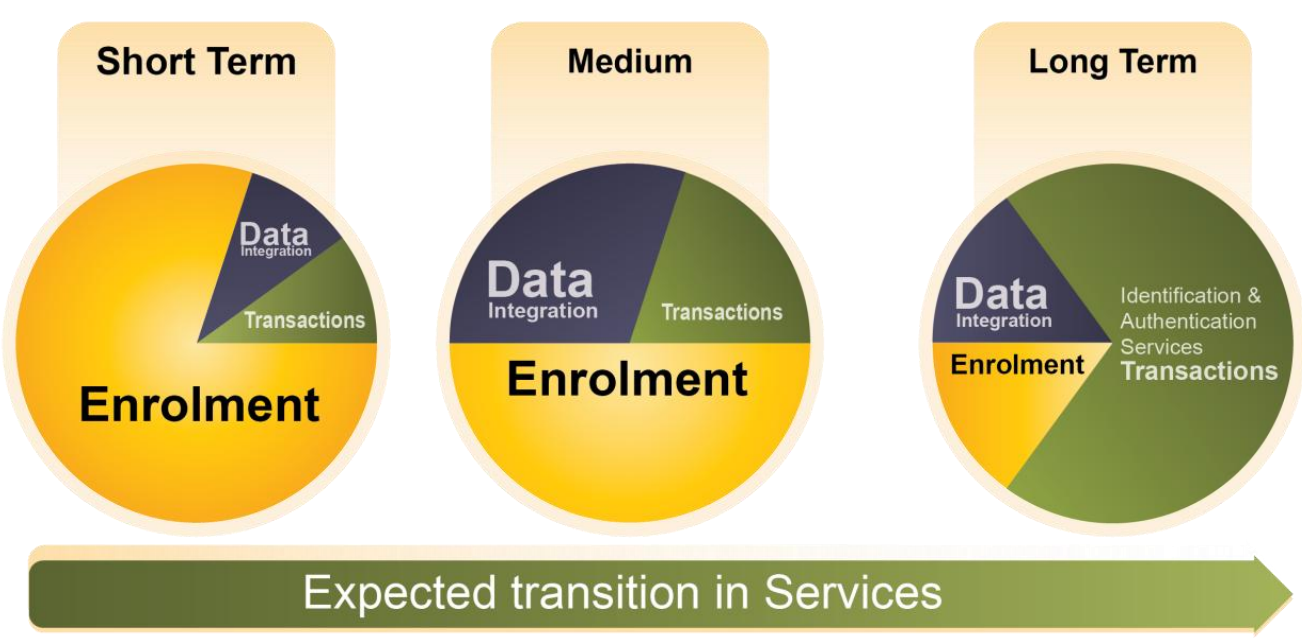

Figure 2. Emirates ID operating model

The definition of the strategy followed a systematic and formal strategy development process that was formulated by the UAE prime minister's office and mandated for use by all UAE federal government entities. The process defined various criteria and controls to ensure integration and consistency across activities and projects as well as the achievement of goals and objectives. The phases of strategy development included a series of key controls:

- Compliance of Emirates ID's vision with the vision and strategy of the federal government;

- Establishing Emirates ID's mission based on its vision;

- Identifying organizational values to support the achievement of Emirates ID's objectives;

- Determining strategic objectives;

- Developing a number of initiatives and programs to meet the targeted objectives;

- Defining strategic performance indicators for initiatives and programs;

- Setting projects to implement the adopted initiatives and programs;

- Distributing projects across Emirates ID's organizational units; and

- Setting operational performance indicators for projects.

The above controls translated into the 2010-2013 strategy map depicted in Figure 3. Strategic initiatives were geared toward the achievement of the strategic objectives as well as the implementation plans for said initiatives, with an eye toward the capacity to adequately measure performance on all fronts by means of operational and strategic key performance indicators. 


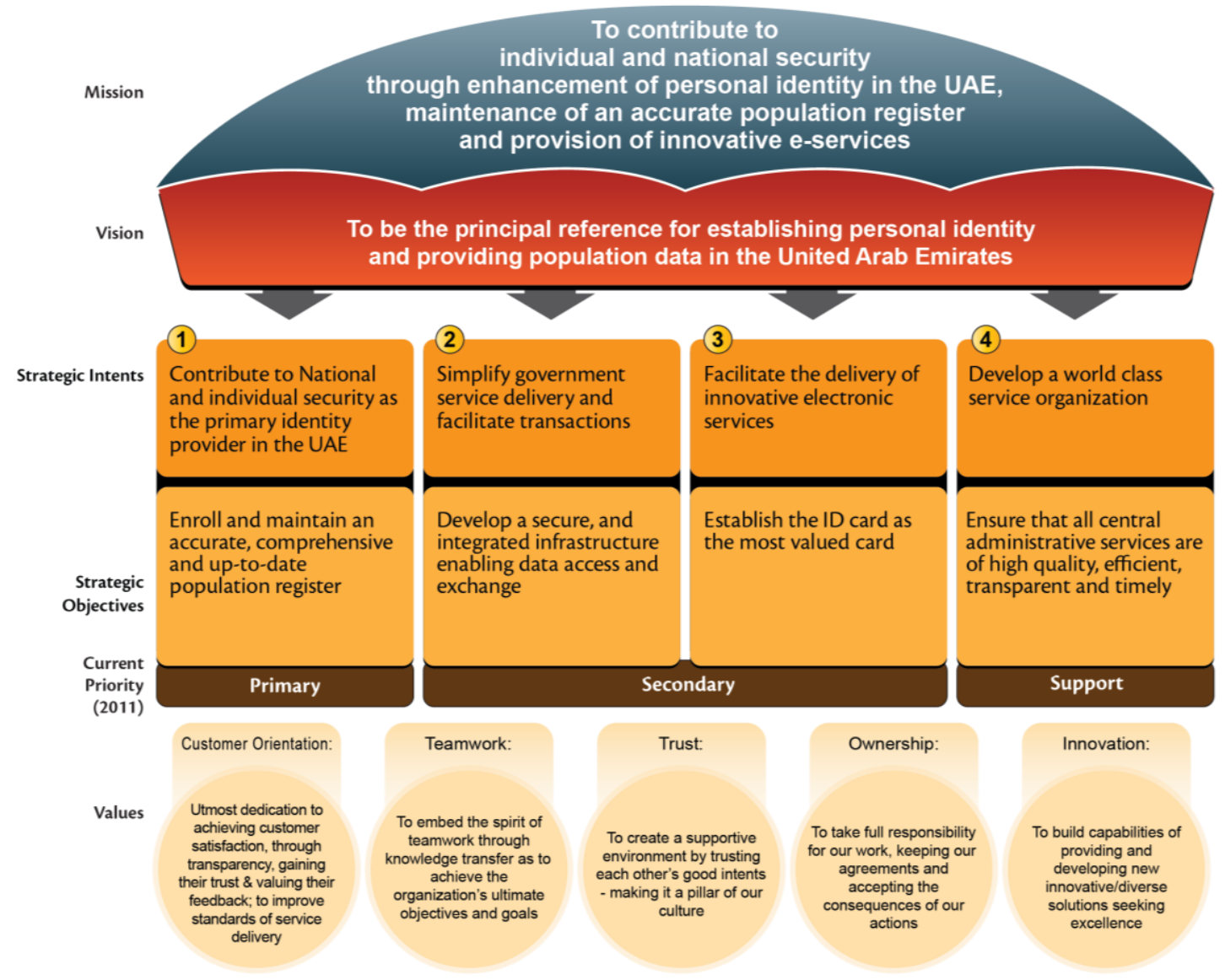

Figure 3. Emirates ID 2010-2013 strategy map

A great amount of attention was given to the development of the strategy map that was later used as a communication medium across the organization. The simplicity of its content and illustration contributed to first shortening the circulation time and, hence, the internal and external awareness of the new strategy and second a holistic view for all departments and units of the overall organizational objectives, leading them to concentrate on outcomes, rather than outputs. In the following sections, we will provide a short overview of the progress made in each of the four strategy objectives.

\section{Strategy Objectives Execution}

\subsection{Objective 1: Enroll and Maintain an Accurate, Comprehensive, and Up-To-Date Population Register}

Achieving Emirates ID's objective of enrolling the UAE population and maintaining an accurate, comprehensive, and updated population register required the completion of some key activities; i.e., re-engineering registration processes fifth, maximizing the registration center's capacity to better manage the flow of customers, and maintaining the accuracy of recorded data. The key success factors involved in enrolling and maintaining an accurate, comprehensive, and up-to-date population register were determined to be an upgrade of the registration process, the timely printing of ID cards, and the ability to manage the flow of customers, as well as the accuracy of data.

The attainment of these success factors was bolstered by the introduction of strategic initiatives, the introduction of key performance indicators geared toward the measurement of the initiatives involved, as well as strategic key performance indicators designed to assess the overall progress in terms of the objective itself. This objective was determined to be the primary priority following a 2011 revision of Emirates ID's strategy. See the Appendices section for more details on some measured and analyzed key performance indicators (KPIs).

\subsection{Objective 2: Develop a Secure and Integrated Infrastructure Enabling Data Access and Exchange}

To develop a secure and integrated infrastructure, Emirates ID focused on the development of interfaces to interact with other entities. Emirates ID also developed the ability to manage the flow of data as well as apply 
rigorous security protocols to ensure protection and safe access to its data. These steps were seen to be key success factors in the achievement of the objective at large.

The progress of this objective was monitored by indicators (shown in the Appendices section) that were designed to determine how secure the authority's infrastructure was in terms of data sharing as well as how satisfied the authority's external partners were with current data-sharing options.

\subsection{Objective 3: Establish the ID Card as the Most Valued Card for Government and Individual Customers}

In order to gauge whether the ID card is, indeed, the most valued card in the UAE, Emirates ID planned to not only embed high added-value services that could be accessed by customers using the information stored on the card (or on the information database) but to also rate its value as a card, based on the number of daily transactions that potential card users may conduct. To deliver this strategic objective, Emirates ID focused upon expanding its partner base (with an initial emphasis on the government sphere with a later inclusion of private sector companies, as well) to adopt the card and promote usability.

The authority liaised with its partners to develop world-class e-government services that were informed by an e-services delivery model framework developed in the UAE. The authority also published its first service catalog to help individual, government, and private sector customers understand what services are available to them and how to access them in a demonstrable bid to increase the awareness of the various functions of the ID card.

5.4 Objective 4: Ensure that All Internal and External Functions and Services Are Customer Focused, Efficient, and Effective

Many of Emirates ID's endeavors concentrated on enhancing its employees' understanding of its strategic goals, specifically toward improved responsiveness to customer needs and higher-quality services. The authority has built a service-based organization that is focused on attaining objectives; improving customer service; and establishing efficient, results-oriented internal and external services. Emirates ID also promoted a focus on performance monitoring, competency development, and the provision of the best possible services.

By enhancing, as well as both proactively and reactively acting upon its feedback channels, offering its people sufficient training to provide world-class services, and tailoring said services to cater to specific customer segments, Emirates ID geared itself toward providing an optimal level of value to its customers. With a focus on achieving excellence, Emirates ID adopted initiatives that championed innovation. These initiatives were designed with an eye toward aiding the authority in building and fostering an organizational culture that embraces a forward-looking mindset and promotes empowerment across the organization's staff.

\section{Strategy Impact on the Organization}

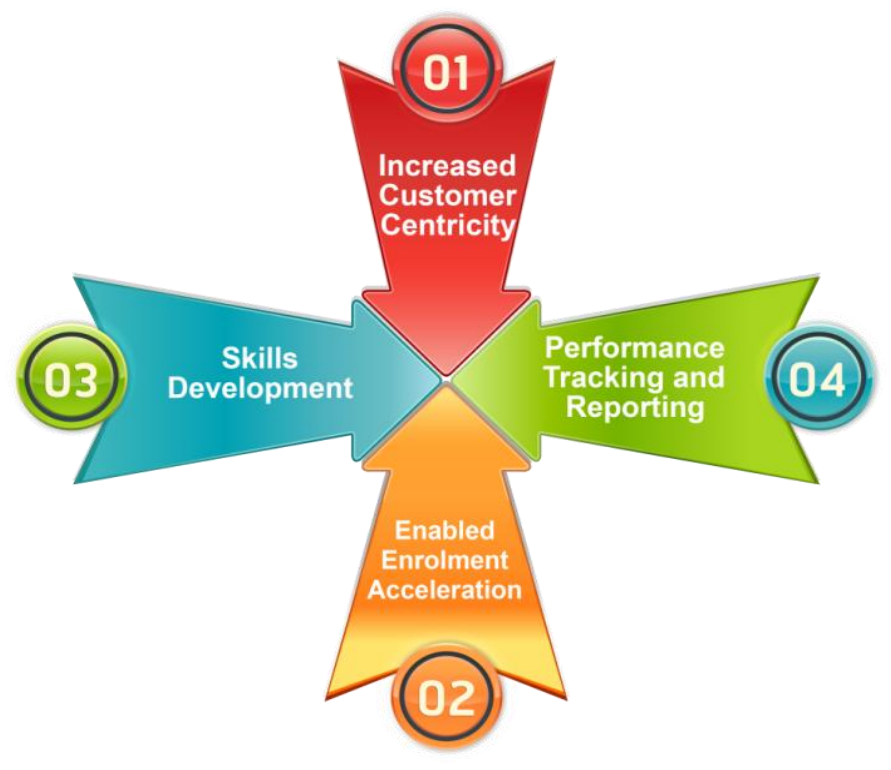

Figure 4. 2010-2013 strategy impact on performance 
The new strategy had a tangible positive impact on the authority's overall performance. (See also Figure 4.) This was visible not only in improved performance indicator results but also in terms of greater leadership, team collaboration, and employee engagement in adopting the new strategy. Below are some key areas that we determined to have been positively affected:

\subsection{Enabled Enrollment Acceleration}

The new strategy acted as an enabler for the acceleration of enrollment rates by facilitating the implementation of reengineered enrollment processes and active monitoring of enrollment processes. There was an:

- Increase in the population register from fewer than 1 million to more than 8 million. This also meant an eight-fold upsurge compared to the earlier period of operation; and

- Increase in the average daily enrollment from under $5 \mathrm{k} /$ day to more than $20 \mathrm{k} /$ day.

\subsection{Performance Tracking and Reporting}

Regular monitoring and internal-external reporting of key performance indicators contributed to the following:

- Improvement in KPI results and a more prevalent culture of accountability for KPI results;

- Emphasis on the monitoring of capacity utilization rates at registration centers; and

- Elimination of root causes/drivers for bottlenecks across enrollment business process steps.

\subsection{Increased Customer Centricity}

The organization paid particular attention to the development of a customer-centric culture. In fact, all of its initiatives were primarily driven by customer needs and wants. As such, Emirates ID culture promoted:

- Ongoing efforts to monitor and improve customer experience; and

- Periodical streamlining of enrollment and card delivery procedures and enhancement of existing and introduction of new customer touch points.

\subsection{Skills Development}

The new strategy played a key role in pushing and accelerating organizational efforts in the development of technical and soft skills of the strategy of the department team and department project managers. These skill sets involved the following:

- Taking an unstructured problem and selecting appropriate information to analyze the issues while keeping in mind the final objectives and not succumbing to micromanagement;

- Independently developing and applying a well-thought-out, comprehensive plan;

- Conducting research that has predefined objectives, is gathered by leveraging appropriate secondary sources, and is supported by meaningful data;

- Adeptness at quantitative and qualitative analytical techniques;

- Rapidly executing project tasks by focusing on core issues and effectively managing deadlines with limited guidance; and

- Summarizing/synthesizing relevant findings and implications into well-structured presentations.

\section{Lessons Learned}

Key factors that contributed to overall success at Emirates Identity Authority were leadership commitment, vigorous and dynamic management mindset, clear visualization of expected outcomes, simplified decision models based on facts rather than feelings, constant communication, and periodical reviews of our plans to adjust them to changing internal and external environments (Al-Khouri, 2012b).

While the planning and execution of the Emirates ID 2010-2013 strategy generated outstanding accomplishments and results, the team also faced some functional and technical challenges. These served as learning points and inputs for the formulation and execution of its later (2014-2016) strategy. These were largely segmented into the following areas: strategy execution, performance management, and strategic vs. operational governance. In each of these areas, Emirates ID encountered challenges from which lessons were learned. Figure 5 provides a summary of these lessons, which are discussed next. 


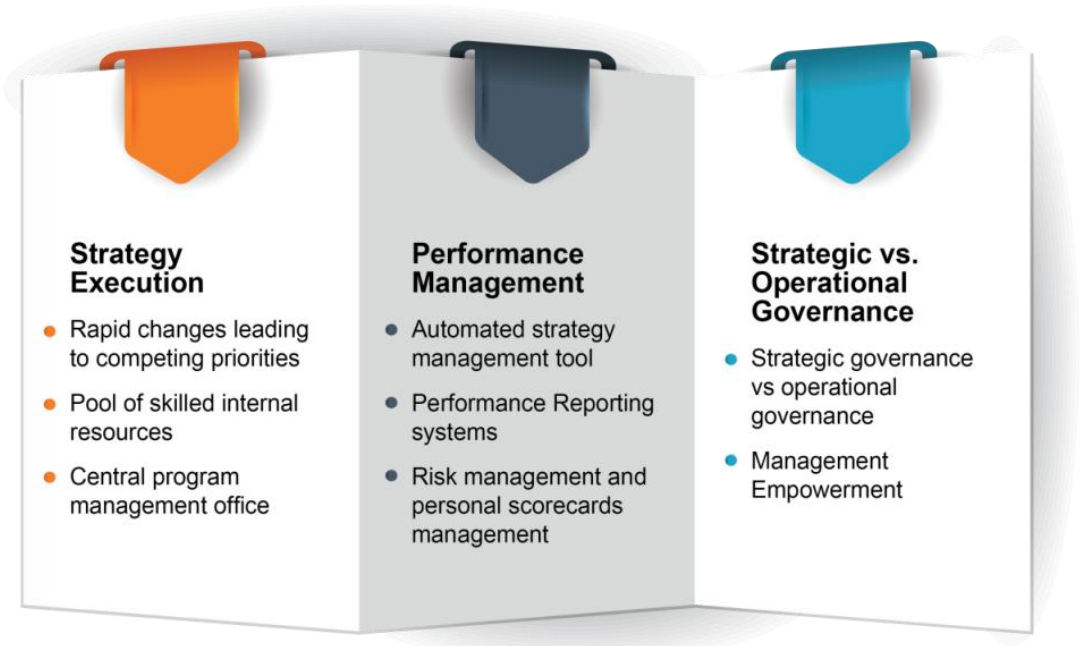

Figure 5. Key lessons from Emirates ID's strategy implementation

\subsection{Strategy Execution}

\subsubsection{Challenge}

The limited pool of internal resources available for the proper execution of all strategic initiatives defined in the 2010-2013 strategic plan was a notable challenge for Emirates ID. This limitation led to the small number of available skilled resources being stretched across too many initiatives, consequentially leading to a decrease in the resources' engagement level and output.

Although a key accomplishment for Emirates ID has been the immense pace at which it has rolled out its key initiatives to close gaps identified during the strategy development phase, this approach has also had an indirect downside: adoption of such a rapid change has led to a lack of prioritization, thereby leading to competing priorities for many parts of the organization and the incomplete implementation of some initiatives.

Underlying drivers for this have been the limited adherence by management to predefined project plans, calling for the phased rollout of initiatives, and the lack of a central program management office that is responsible for maintaining an integrated master plan and enforcing disciplined issue-management processes and standardized project reporting.

\subsubsection{Solution}

Action plans incorporated a new approach to ensure that all strategic initiatives are properly funded and supported by teams with the right competencies and skill sets to manage and execute them. The high-level strategic initiatives initially defined by the leadership team were assigned to sector and departmental executives, who were given the responsibility for translating the initiatives into specific projects with detailed implementation plans, budget estimation, the identification of competencies required to execute them, and the definition of the expected (quantifiable) impact each project would bring to corporate targets and objectives.

After all of the initiatives were described, a second prioritization process took place in order to define an integrated roadmap based on the availability of resources (both human and financial) and a robust change management plan to incorporate the missing competencies and skills of the internal team. Finally, projects were incorporated into the operational plans of each department and accountability for the execution and reporting was assigned to the department managers.

\subsection{Performance Management}

\subsubsection{Challenge}

During the development of the strategy, a detailed assessment on management processes was performed throughout the organization. A common need was identified: a performance management and reporting system to support the decision-making process at all organizational levels. The absence of an automated strategy management tool to measure and report on strategy performance resulted in various forms of inefficiencies and inconsistencies during the 2010-2013 timeframe. 
Reporting was limited to Excel and PowerPoint, and the time spent on manual data collection and consolidation processes was time that should have, in theory, been dedicated to performance analysis. Risk management and personal scorecard management were also identified as additional functionalities that would be required in the near future.

\subsubsection{Solution}

To tackle this issue, a well-structured process was followed to identify detailed business, technical, and functional requirements and select vendors for the solution. After several rounds of product demonstrations from the shortlisted vendors, a "vendor's scorecard" was developed and software was selected. The chosen solution derived decision making at all organizational levels, taking the organization to the next step of strategy execution where each employee was able to visualize his contribution to the corporate strategy using an integrated, online, cloud-based platform.

\subsection{Strategic vs. Operational Governance}

\subsubsection{Challenge}

Another challenge faced during the execution of the strategy was the time consumed by the senior management team in the identification and implementation of solutions for tactical and/or operational issues, thereby "distracting" the focus from strategic governance to operational governance-leading to an overburdened and singular steering committee (through weekly management team meetings) that maintained oversight across almost all initiatives.

\subsubsection{Solution}

To prevent such situations, the mid-management team was asked to take on a more proactive, independent role in issue identification and resolution, whereas the senior management team would ensure that these individuals would be empowered to make decisions and given room to learn from their mistakes. The governance process was redesigned, as described above, to facilitate this process, empowering the different management levels to recommend solutions and take actions regarding operational issues during the weekly management meetings, while strategic issues were discussed during the quarterly strategy review meetings.

\section{Concluding Remarks}

Governments' strategies normally tend to be clear, to some extent, regarding their vision and high-level outcomes when they are first developed; however, our experience indicates that many formulated government strategies do not capture or take into account the complexity or nature of these strategies at the execution stage. As a result, they face significant difficulties in articulating their requirements in terms of clear objectives, actionable initiatives, and precise and measurable performance indicators. In fact, strategies in governments, due to their multifaceted nature, face a greater risk of failure than those in the private sector. We need to always remember that complexity can easily destroy the value we intend to bring through our strategies. It should, therefore, be the goal of every organization to decrease complexity wherever and whenever deemed possible.

The strategy implementation in the researched organization, as shown in this article, yielded successful outcomes after 4 years of well-planned and disciplined execution. The outlined case study illustrates the need to focus on adding value beginning with strategy development and during execution. However, it is also important to remember that there will never be a "one-size-fits-all" recipe for success; nevertheless, learning from others' experiences may feed existing limited knowledge pools, especially from some of the untapped explorations. Our case study has, at least, a few things to offer!

\section{References}

Al-Khouri, A. M. (2007). Using Quality Models to Evaluate National ID systems: the Case of the UAE. International Journal of Social Sciences, 1(2), 117-130.

Al-Khouri, A. M. (2012b). Targeting Results: Lessons Learned from the UAE National ID Program. Global Journal of Computer Application and Technology, 2(1), 830-836.

Al-Khouri, A. M. (2014). Fusing Knowledge Management into Public Sector Corporates Excellence Culture: A Review of the Field and The Case of Emirates Identity Authority. Journal of Knowledge Management, Economics and Information Technology, 4(3), 1-60.

Carroll, P, B., \& Mui, C. (2008). Failed Strategies. What CEO's can learn from the billion dollar mistakes. Chief Executive, (236), 44-46.

Eisenhardt, K. M. (1999). Strategy as Strategic Decision Making. Sloan Management Review, 40(3), 65-72. 
Skinner, W. (1969). Manufacturing - Missing Link in Corporate Strategy. Harvard Business Review, May-June 1969.

\section{Appendix}

Appendix 1. Measured and analyzed KPIs
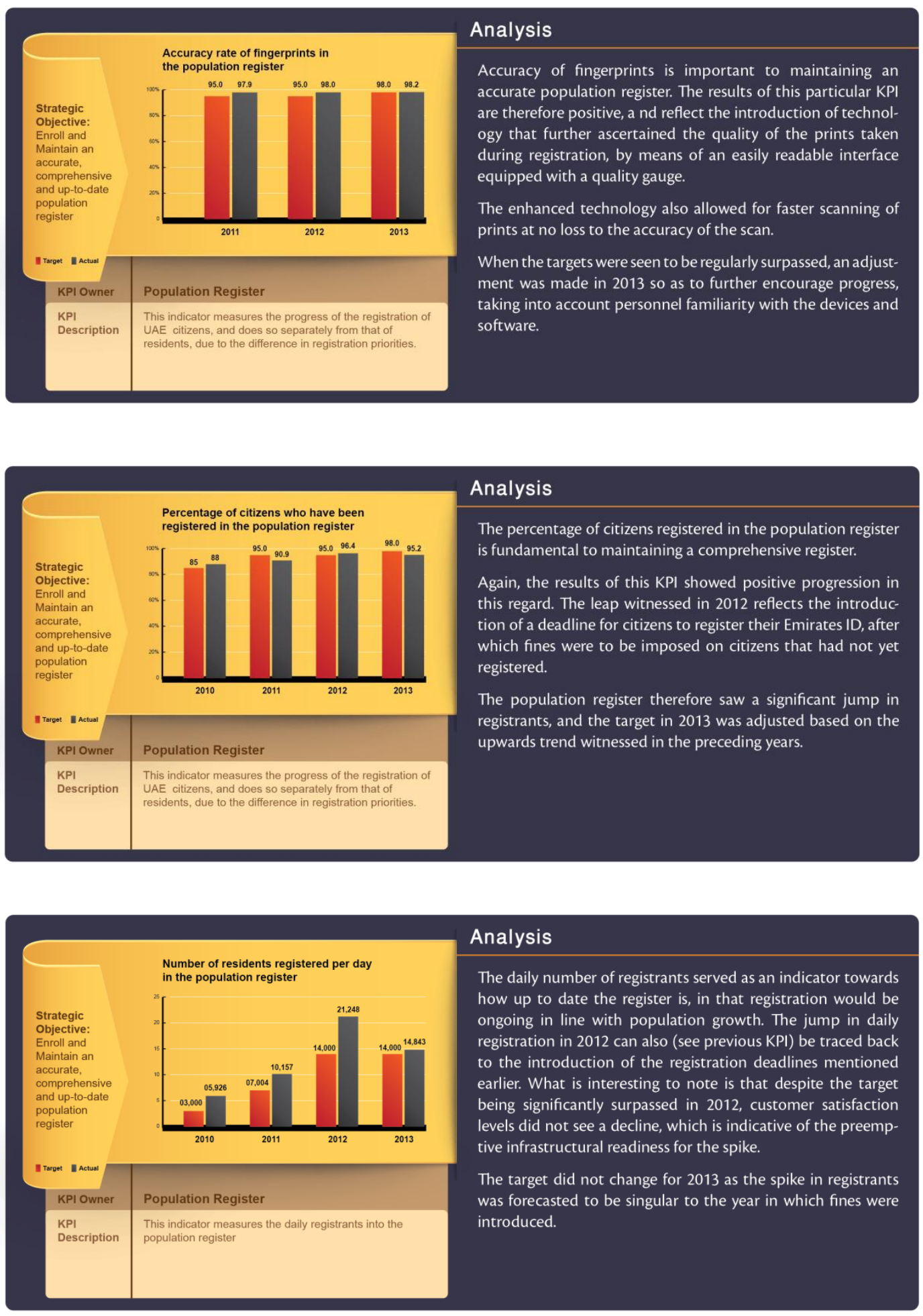

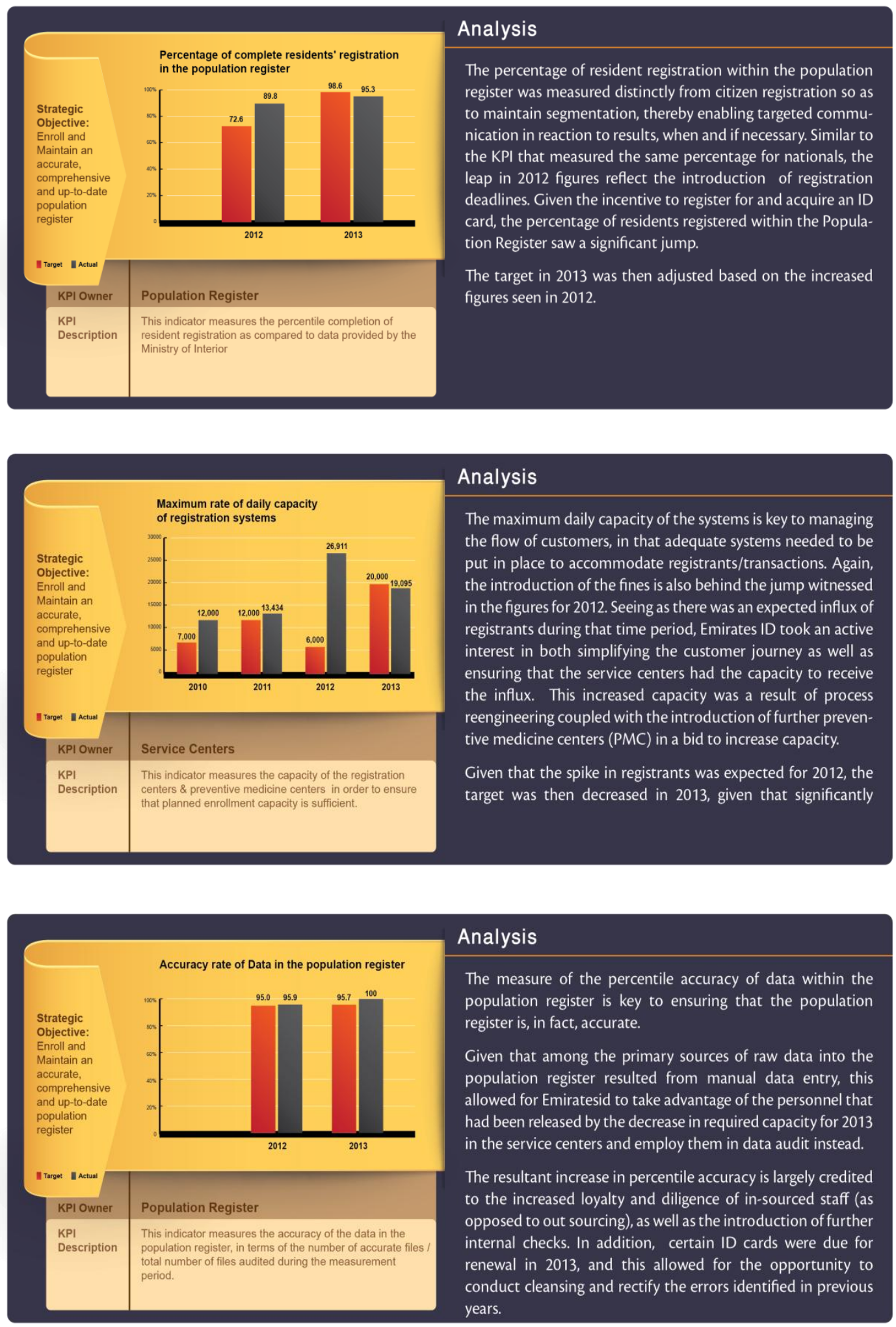
Appendix 2. Measured and analyzed KPIs
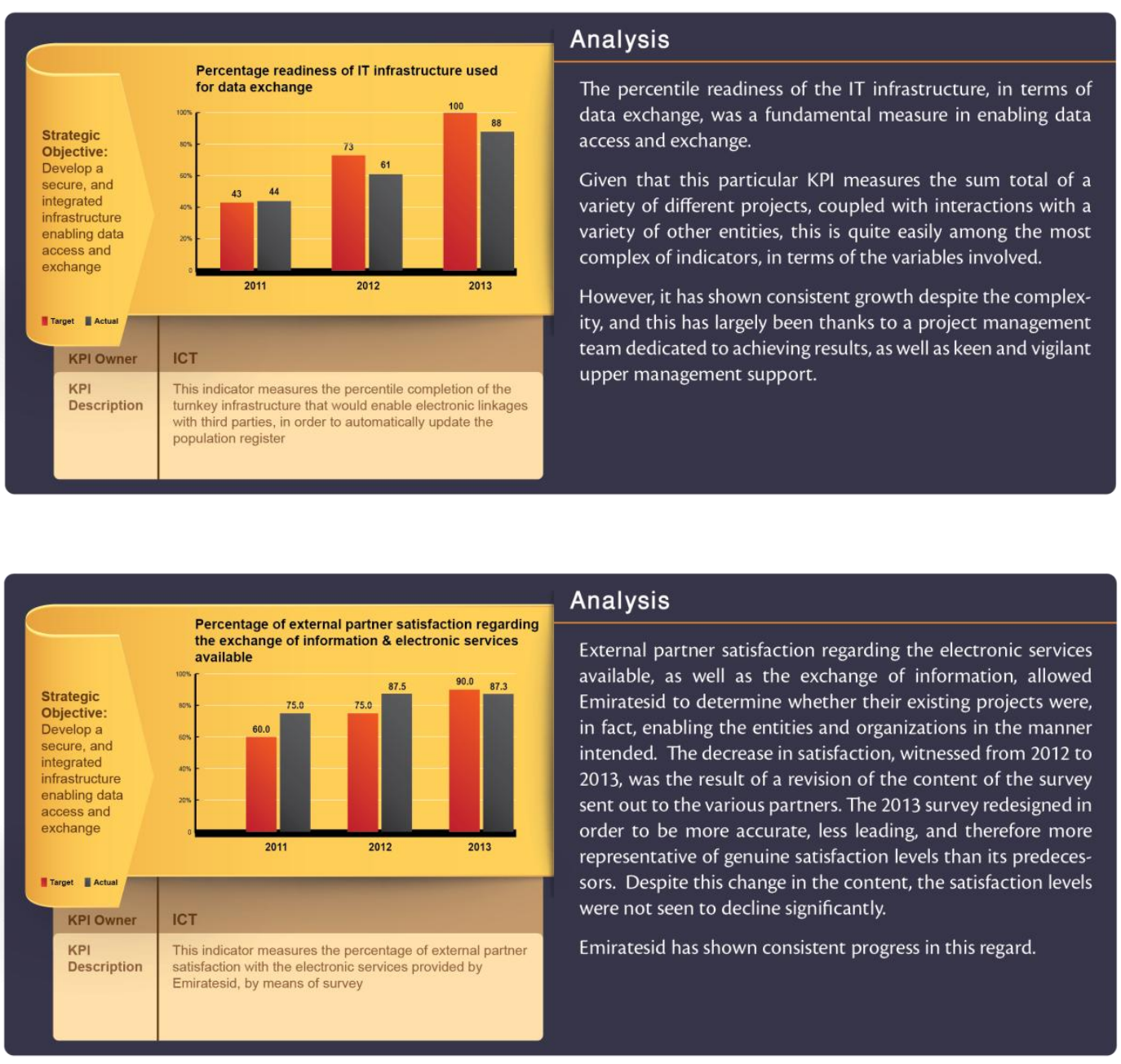

Appendix 3. Measured and analyzed KPIs

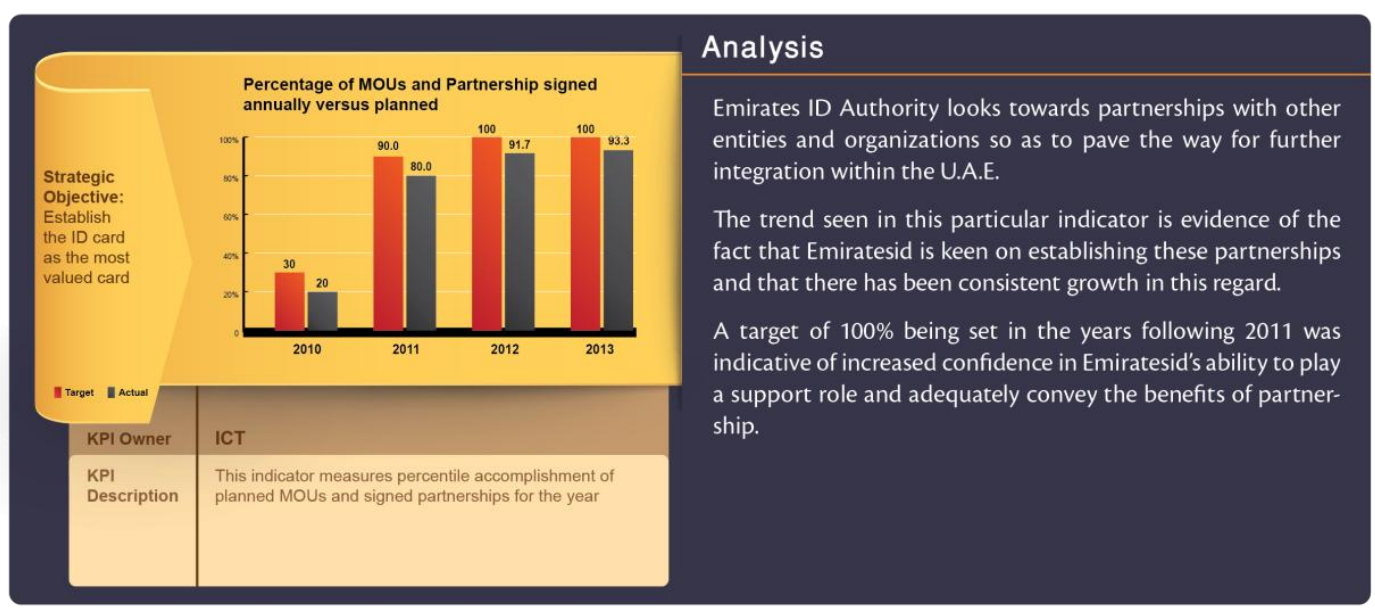




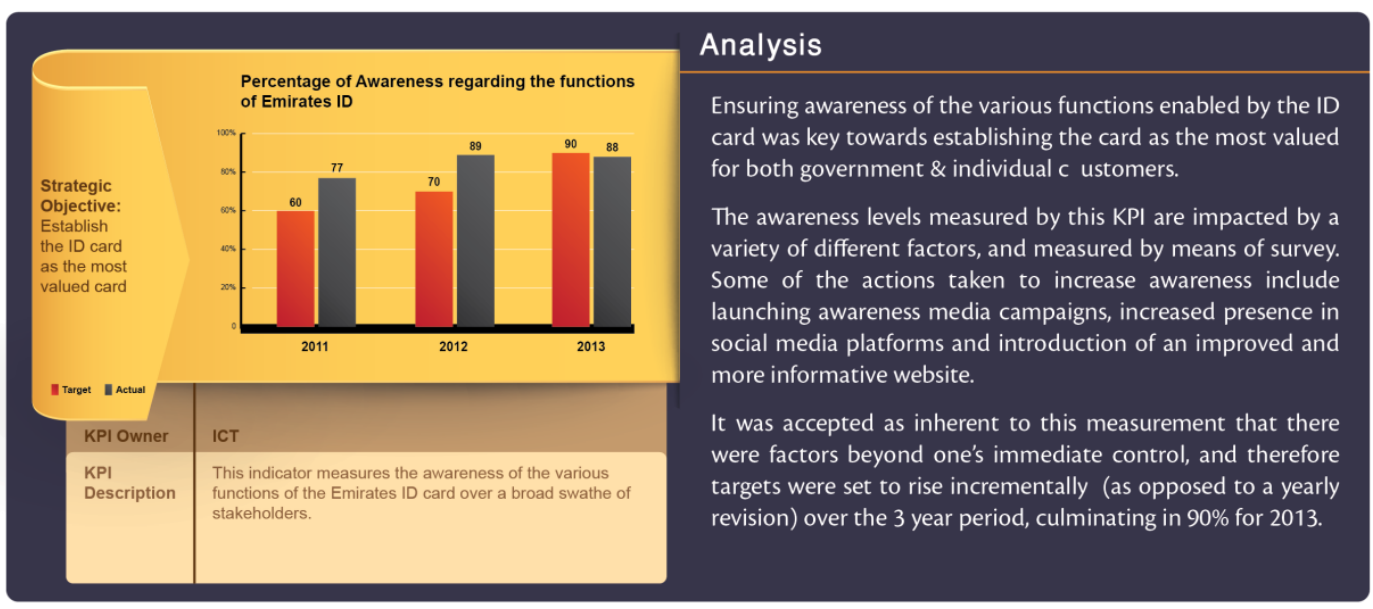

Appendix 4. Measured and analyzed KPIs
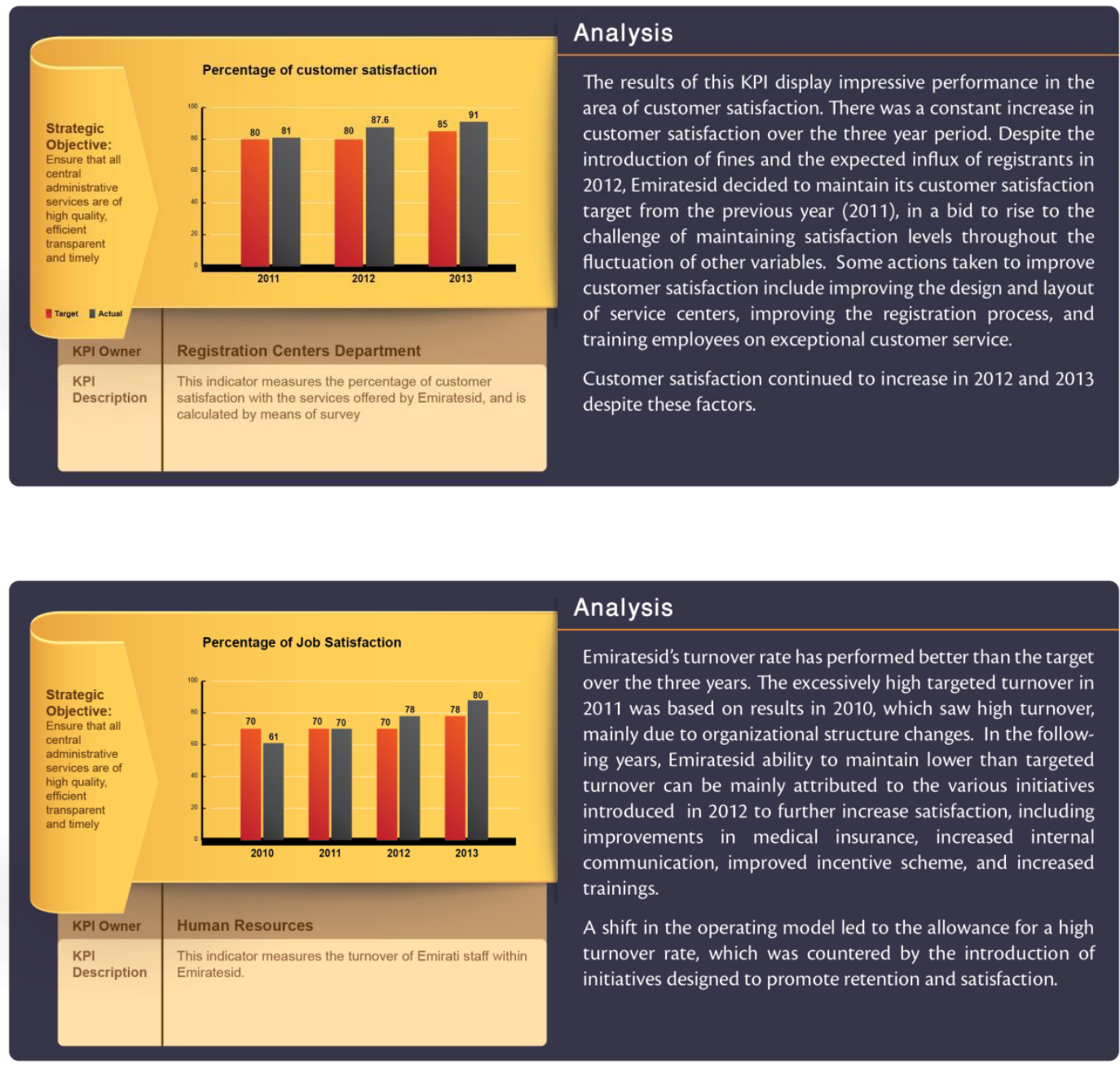

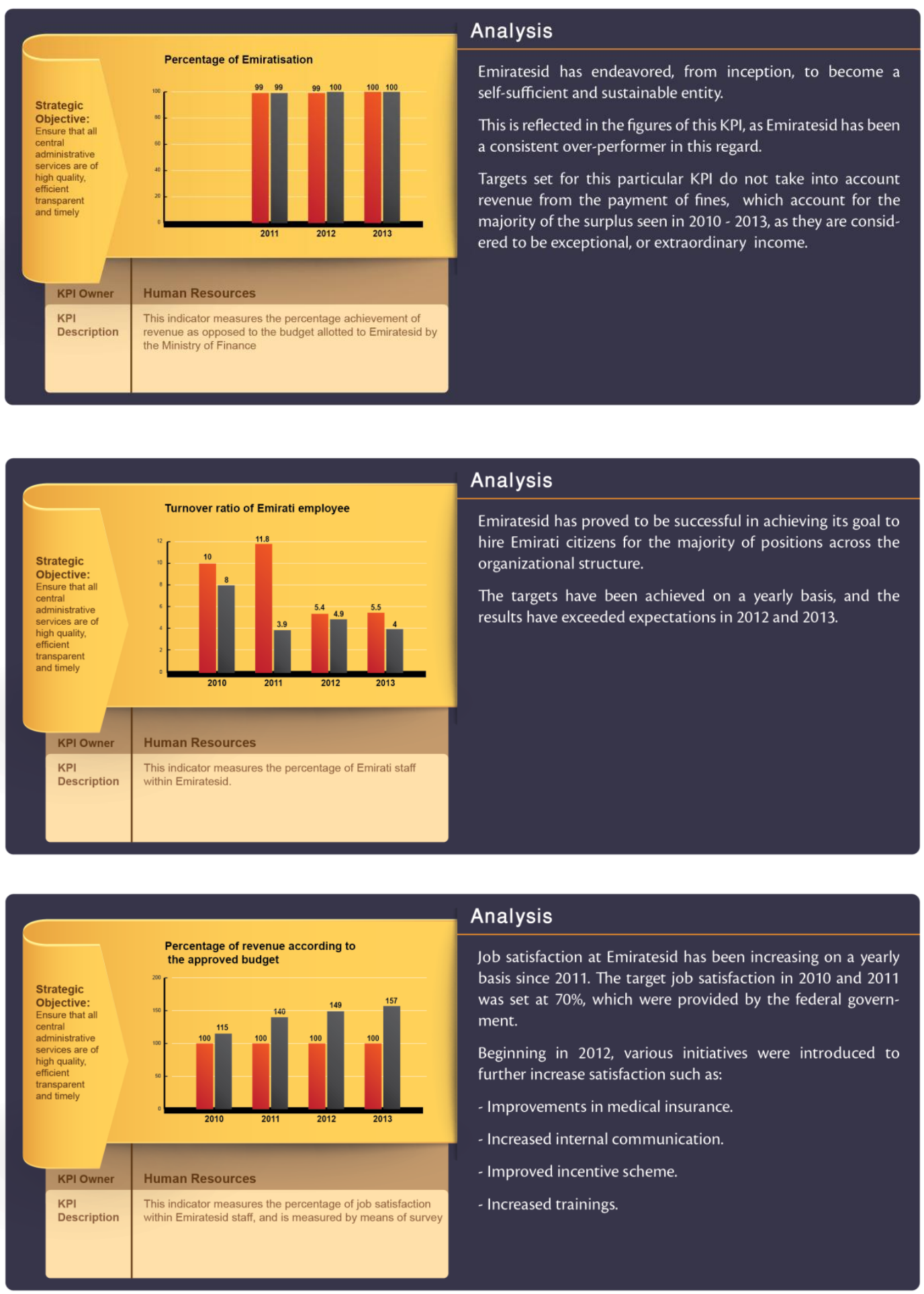

\section{Copyrights}

Copyright for this article is retained by the author(s), with first publication rights granted to the journal.

This is an open-access article distributed under the terms and conditions of the Creative Commons Attribution license (http://creativecommons.org/licenses/by/3.0/). 